\title{
Ein Konzept zur Mobilisierung einer kritischen Masse anhand der geschichtsphilosophischen Überlegungen von Walter Benjamin
}

\begin{abstract}
Rafael Rehm*
Zusammenfassung

Im folgenden Artikel soll anhand der geschichtsphilosophischen Überlegungen von Walter Benjamin ein alternatives Konzept zum Habermasschen Verzicht auf eine Geschichtsphilosophie überhaupt herausgearbeitet werden, welches es erlaubt, historische Prozesse und somit die Bilder der Lebenswelt als Metaerzählung neu zu chiffrieren, um somit den Begriff des gesellschaftlichen Fortschritts neu zu verorten und ihn für die Mobilisierung einer kritischen Masse brauchbar zu machen. Es geht darum, eine Dialektik im gegenwärtigen Diskurs zu positionieren, die sich sowohl von einer geschichtspositivistischen Hegellektüre als auch von einer postmodernen Resignation unterscheidet. Im Weiteren geht es darum zu zeigen, warum eine solche Dialektik, die notwendigerweise zugleich Geschichtsphilosophie ist, für die Bedingung der Möglichkeit der Mobilisierung einer kritischen Masse konstitutiv ist.
\end{abstract}

Schlagwörter: Geschichtsphilosophie, Walter Benjamin, Dialektik, kritische Masse

\section{A concept for the mobilization of a critical mass on the basis of Walter Benjamin's philosophy of history}

Abstract

The article aims to depict the epistemological program of Walter Benjamin in terms of his concept of a knowledge of history. The intention of this depiction is to elaborate a method that makes an alternative reading of historic processes possible. Besides this, the intention is to question the difference between Walter Benjamin's conception and Hegel's conception of knowledge of history. In this formulation of a question the differences between the dialectic of Hegel and Benjamin's conception of it will be discussed. The alternative reading of Walter Benjamin which makes a materialistic interpretation of the present time possible and helps to mobilize a critical mass will be questioned and discussed in the following article.

Keywords: philosophy of history, Walter Benjamin, dialectic, critical mass

\footnotetext{
* Rafael Rehm, Universität Jena. E-Mail: rr.rafaelrehm@gmail.com
} 


\section{Einleitung}

„Nein, in diesen beschränkten, konservativen und feigen Köpfen findet unser Zeitalter keinen Raum"(Trotzki 1935/1962: 108).

Am 21. Januar 2017, einen Tag nach dem Antritt von Donald Trump, dem 45. Präsidenten der Vereinigten Staaten, begingen knapp 700.00o Menschen den sogenannten Women's March on Washington. Den Organisatorinnen und Organisatoren des Women's March ging es nach eigenen Angaben darum, sich aktiv für Frauenrechte einzusetzen. ${ }^{1}$ Darüber hinaus gab es keine konkreten Forderungen. Bis heute treffen sich seit dem 19. Dezember 2014 jeden Montag einige hundert Menschen im Rahmen der sogenannten PEGIDA-Demonstrationen in Dresden, um vor einer Islamisierung des Abendlandes zu warnen. Beide Gruppen stehen, nach ihrem eigenen Verständnis, für den Schutz demokratischer Grundrechte und die Interessen derer ein, die sie zu repräsentieren beanspruchen. Es scheint in unserer Zeit ein Trend $\mathrm{zu}$ sein, gegen das Establishment $\mathrm{zu}$ mobilisieren, und dies, sowohl von linker als auch von rechter Seite aus, mit den Methoden einer Identitätspolitik. Vertreterinnen und Vertreter diverser Bewegungen stehen nach eigener Angabe für die Rechte bestimmter Gruppen ein. Was die meisten Protestbewegungen eint, ist der Glaube daran, dass die Möglichkeit für einen Fortschritt im Sinne einer Verbesserung der Lebensbedingungen, gegeben ist. Diese Vorstellung ist daran gekoppelt, dass angenommen wird, dass der positive Appell einer Umsetzung bestimmter Werte (wie beispielsweise Freiheit oder nationale Selbstbestimmung) an die Repräsentantinnen und Repräsentanten bestimmter Interessengemeinschaften dazu beiträgt, einen Fortschritt innerhalb des bestehenden Systems zu garantieren. Eine Masse, die diese Ziele hat, macht lediglich darauf aufmerksam, dass sie es für möglich hält, besser regiert bzw. besser beherrscht zu werden. Macht sie das aber schon zu einer kritischen

1 Cassady Fendlay, eine Organisatorin des Women's March on Washington, sagt im Artikel von Amber Jamieson „Women's March on Washington: a guide to the post-inaugural social justice event" im Guardian vom 27. Dezember 2016: „The Women's March on Washington is quick to say it is not an anti-Trump protest. ,We're not targeting Trump specifically. It's much more about being proactive about women's rights,' said Cassady Fendlay, spokeswoman for the march." Online: https://www.theguardian.com/us-news/2016/dec/27/ womens-march-on-washington-dc-guide [17.02.2017]
Masse? Und wenn nein, wie mobilisiert man angesichts diverser Bewegungen von Massen eine kritische Masse und worin liegt ihre spezifische Differenz zu anderen Massen? Kann man überhaupt eine kritische Masse mobilisieren oder von einer kritischen Masse sprechen, die nicht an den Fortschritt glaubt und keine positiven Appelle mehr an irgendjemanden richtet? Ziel ist es, dass diese Fragen nach und nach in diesem Aufsatz beantwortet werden können.

„Fortschritt heißt: aus dem Bann heraustreten, auch aus dem des Fortschritts [...]“ (Adorno 1975/2016: 156). Der Anspruch, welchen Adorno in diesem Zitat geltend macht, liegt darin, dass das, was als gesellschaftlicher Fortschritt im Sinne einer Verbesserung der Lebensverhältnisse bezeichnet wird, bloß das Fortschreiten der Ausbeutung bildet, aus dem es auszubrechen gilt. Die Genügsamkeit attestiert der Gegenwart die Legitimation für die in ihr konkret existierenden Verhältnisse. Der Blick auf eine ruhmreiche Vergangenheit hat scheinbar keine Ambitionen auf einen Bruch mit der Gegenwart mehr. Die notwendige Erkenntnis, dass das bloße Fortschreiten der technischen Entwicklung nicht notwendigerweise einen Fortschritt zu einer besseren, humaneren und gerechteren Menschheit bedeutet, scheint ein alter Hut und der Überrest dessen, was man mit der sogenannten ersten Generation der Frankfurter Schule und ihrer Vertreter identifizierte.

Geht es denn überhaupt anders? Kann man überhaupt, gemäß der Definition des Fortschritts nach Adorno, aus dem Bann heraustreten, der den Fortschritt selbst ausmacht ohne der absoluten Resignation und Handlungsunfähigkeit zu verfallen? Diese Frage möchte ich im Rahmen dieses Aufsatzes im Hinblick auf die Mobilisierung einer kritischen Masse mit Hilfe der geschichtsphilosophischen Überlegungen nach Walter Benjamin bedenken. Hierfür werde ich erstens klären wie ich eine kritische Masse verstehe, zweitens werde ich am Modell der Hegelschen Geschichtsphilosophie die Problematik eines Fortschrittsdenkens explizieren. Drittens werde ich darstellen, wie die Mobilisierung einer kritischen Masse mit Hilfe einer Geschichtsphilosophie nach Walter Benjamin verstanden werden könnte. Hierfür greife ich auf einige Schlüsselzitate aus Über den Begriff der Geschichte (1940) und aus dem Konvolut N [Erkenntnistheoretisches, Theorie des Fortschritts] aus dem Passagen-Werk zurück. Der Fokus liegt hierbei auf der Methodik, welche die Mobilisierung einer kritischen Masse jenseits des Fortschrittsparadigmas denkbar machen soll. Hierfür werde ich dann auch konkrete Beispiele aus dem Euvre Benjamins zu 
Hilfe ziehen. Viertens werde ich ein Fazit ziehen und auf die offenen Fragen der aufgeworfenen Ideen eingehen.

\section{Was ist eine kritische Masse?}

Grundsätzlich gilt es am Anfang dieses Aufsatzes den Begriff einer kritischen Masse, wie er hier zur Anwendung kommen soll, hinreichend zu klären. Im Rahmen dieser Arbeit verstehe ich den Begriff einer kritischen Masse als einen Ort politischer und oppositioneller Macht. Im Idealfall zeichnet sich eine kritische Masse durch zwei Merkmale aus. Erstens ist eine kritische Masse fähig zur politischen Handlung, und zweitens benötigt eine kritische Masse hierfür gewisse synchrone Handlungsweisen. Spätestens seit Elias Canettis Werk Masse und Macht ist deutlich geworden, welche Bedrohung die Bildung von Massen mit sich bringt, denn verfestigt sich eine Masse $\mathrm{zu}$ einem Gebilde allgemeiner Identität, so droht in ihr das Individuum und mit ihm jeder Anspruch auf Humanität unterzugehen (vgl. Canetti 1994). Selbst eine kritische Masse droht, herrscht in ihr erst einmal der Zwang zur Homogenisierung, sich hin zum totalitären Konstrukt zu entwickeln. Auch Bewegungen wie die der NSDAP haben in der scheinbaren Bildung oppositioneller und kritischer Massen ihren Ursprung. Ich sehe also gerade den Begriff der Kritik als notwendige Bedingung für eine Masse, nicht einem totalitären Charakter zu unterliegen. Im Rahmen dieses Aufsatzes verstehe ich Kritik im Sinne der kritischen Theorie als Ideologiekritik. Der Begriff der Kritik im Sinne Horkheimers geht in der Darstellung in seinem Essay aus dem Jahr 1937 Traditionelle und kritische Theorie über die kategorialen Bestimmungen der Gesellschaft hinaus. Dies bedeutet, er bewegt sich jenseits der Grenzen dessen, was eine Gesellschaft als den Rahmen für den Gegenstand der Kritik festsetzt, indem er mit der dialektischen Methode der Philosophie eine Kritik der Verflechtung des gesellschaftlichen Ganzen zugesteht (vgl. Horkheimer 1988: 82/84 und 209-216). In diesem Sinne ist sein Begriff von Kritik ideologiekritisch, denn er verweilt nicht bei der Kritik einer einzelnen Bestimmung eines Sachverhalts, sondern geht über zu einer Kritik der geschichtlichen Entstehung gesellschaftlicher Kategorien von Kritik überhaupt und bezieht diese auf die Ökonomie und die gesellschaftliche Arbeitsweise. Das Anliegen einer solchen Kritik ist es, die Konturen eines Marxschen Konzepts des Überbaus im Sinne einer verdinglichten Lebenswelt, hin zu deren Struk- turen und Machtgefüge, ans Licht zu bringen. Kritik impliziert somit immer eine alternative Lesbarkeit des gesellschaftlich-politischen Lebensraums.

Eine kritische Masse, insofern sie kritisch genannt werden will, muss nicht nur Distanz zum gesellschaftlich-politischen Konsens, sondern auch zu sich selbst, als einem von diesem Konsens diskursiv durchdrungenen Geflecht, herstellen, ansonsten unterliegen die emanzipatorischen Kräfte in einer kritischen Masse wiederum totalitären Strukturen. Die Frage ist aber, wie kann ein gesellschaftlich immanenter Teil von Menschen in Distanz zum Gesamtzusammenhang einer Gesellschaft gehen, über die Gesellschaft urteilen und zugleich selbst Teil dieser Gesellschaft sein? Wie kann eine Erkenntnis der gesellschaftlichen Verhältnisse zustande kommen, wenn der Symbol- und Bildraum einer Gesellschaft nicht den Zustand und die Mechanismen der Ausbeutung zum Ausdruck bringt, sondern dieser Ausdruck überblendet wird? Einerseits betont die deutsche Bundesregierung beispielsweise ununterbrochen die Zahl der von der Bundesrepublik aufgenommenen Kriegsflüchtlinge aus Syrien und inszeniert sich somit als Vorreiter in Bezug auf die Umsetzung der Menschenrechte in Zusammenhang mit dem Anspruch der Genfer Konventionen. Andererseits wird aber ein Zusammenhang der wirtschaftlichen Interessen Deutschlands und der EU und dem Schicksal der Menschen in Syrien gekonnt ausgeblendet. ${ }^{2}$ Diverse Nationalstaaten lassen sich als Retter derer feiern, deren Existenz sie zuvor auf der Basis ihrer eigenen ökonomischen Interessen zerstört haben. Die humanitären Katastrophen, die in vielen Fällen gerade auf den Konkurrenzkampf der großen Industriestaaten unter den Produktionsverhältnissen einer kapitalistischen Ökonomie zurückzuführen sind,

2 Die FAZ berichtet am 08.12.15. über die Quellen von Amnesty International und stellt fest: „So seien etwa Sturmgewehre aus Belgien, China und den Vereinigten Staaten im Einsatz. Zudem verwendet der IS laut Amnesty Gewehre aus Deutschland, die von dem Waffenhersteller Heckler \& Koch produziert wurden." (http://www.faz.net/ aktuell/politik/ausland/naher-osten/amnesty-internationalis-kaempfer-schiessen-mit-deutschen-waffen-13954173.html) In der FAZ- Ausgabe vom 16. Oktober 2016 ist nachzulesen, dass sich die Rüstungsexporte der Bundesrepublik im Jahr 2015 auf 7,86 Milliarden Euro beliefen. Im Jahr 2014 betrugen dieselben für das Jahr 2014 3,97 Milliarden Euro. Dies ist der höchste Stand der Bundesrepublik für Rüstungsexporte in diesem Jahrhundert. Online: www.faz.net/aktuell/wirtschaft/ wirtschaftspolitik/waffenindustrie-deutsche-ruestungsexporte-verdoppeln-sich-nahezu-1432156o.html [17.02.2017]. 
werden somit überblendet. Eine kritische Masse muss es sich zur Aufgabe machen, die Pathologien unseres Zeitalters und ihre Zusammenhänge sichtbar $\mathrm{zu}$ machen. Die Methode, um dies zu tun, wird im Laufe dieses Aufsatzes erläutert werden.

\section{Das Dilemma der Hegelschen Auffassung von Geschichte in Bezugnahme auf die Mobilisierung einer kritischen Masse}

Die Frage, die sich hier als Erstes stellt, ist die Frage nach der Aktualität einer Hegelschen Geschichtsphilosophie. Warum greife ich überhaupt auf die Hegelsche Geschichtsphilosophie zurück? Vor allem, wo es doch spätestens seit der Theorie des kommunikativen Handelns von Jürgen Habermas klar zu sein scheint, dass es überhaupt keine Geschichtsphilosophie für das Wesentliche einer Gesellschaftskritik im marxistischen Sinne mehr braucht. ${ }^{3}$ Jedoch geht es weniger um die spezifische Beschaffenheit der Hegelschen Dialektik als um ein zutiefst problematisches Denken, welches eine solche Dialektik in ihrem vulgären Verständnis und in Bezugnahme auf die Mobilisierung einer kritischen Masse impliziert. Dieses vulgäre Verständnis liegt in einer Glorifizierung des Bestehenden als Stadium eines fortschrittlichen Prozesses der Gesellschaft als ganzer begründet. Hegel und sein dialektisches Verständnis der Menschheitsgeschichte scheint zwar im akademischen Diskurs überwunden, aber das kulturelle Selbstverständnis unserer Gesellschaft führt implizit einen Fortschrittsdiskurs weiter, der es nur bedingt erlaubt, gesellschaftliche Mechanismen zu hinterfragen. Die Hauptaufgabe einer kritischen Masse liegt aber genau in einer permanenten In-Frage-Stellung der Legitimationsstrategie gesellschaftlicher Mechanismen, ohne Rücksicht auf das, was man allgemein beansprucht unter den großen Errungenschaften der westlichen Zivilisation $\mathrm{zu}$ verbuchen. Das bedeutet, dass jeder Gesellschaft ihre je eigene Metaerzählung zu Grunde liegt. Grob gesagt gilt in Mitteleuropa bis hinein in die Gegenwart der Vorwand, das Erbe der Aufklärung,

3 Nachdem bereits die frühe kritische Theorie eine Kritik der Hegelschen Dialektik auf Basis der geschichtsphilosophischen Implikationen derselben auf gesellschaftskritische Weise vollzogen hat, ist es die Aufgabe der Habermaschen Konsenstheorie, die normativen Postulate einer kritischen Theorie aufrecht zu erhalten, indem sie sich von der Hegelschen Betrachtungsweise einer Hegelschen Geschichtsphilosophie und infolge dessen von einer Dialektik überhaupt verabschiedet. die Anliegen der Französischen Revolution und die moderne Form der Demokratie durchzusetzen, unter welchem die Geschichte als fortschrittlich begriffen wird. Die europäische Geschichte erscheint unter diesem Aspekt als ein Prozess der Emanzipation, deren stetiger Fortschritt sich in der Explikation menschlicher Freiheit ausdrückt. Die Idee der Freiheit wird zur Legitimation und Begründung des Fortschrittdenkens. Es geht darum, ein Bewusstsein kollektiver und individueller Freiheit durch die Entfaltung eines vernünftigen Denkens stets zu fördern. Leitfaden hierfür ist die Würde des Menschen. Schon Hegel bemerkte in seinen Vorlesungen über die Philosophie der Geschichte: „Die Geschichte ist ein Fortschreiten im Bewusstsein der Freiheit" (Hegel 1821/1969: 32). Das Problem dieser Aussage liegt in einem Selbstverständnis, welches ungeachtet gegenwärtiger humanitärer Katastrophen, wie beispielsweise dem Krieg in Syrien, an einer bestimmten und zentralen Auffassung von Geschichte festhält. Diese Auffassung liegt darin, Geschichte, genauer die Geschichte der Menschheit, als kontinuierlichen Fortschritt zu betrachten. Nicht als bloßes Fortschreiten, sondern vielmehr als Verbesserung des Zustands der gesellschaftlichen Lebenswelt mit jeder Epoche. Weiter heißt es bei Hegel: „Die Philosophie [...] lehrt uns, daß alle Eigenschaften des Geistes nur durch die Freiheit bestehen, alle nur Mittel für die Freiheit sind, alle nur diese suchen und hervorbringen [...] (ebd.)." (ebd.). Mittel für die Freiheit zu sein, gilt letztlich auch für das Individuum. Hierin liegt die Paradoxie. Freiheit ist in diesem Sinne nicht das Vermögen eines Individuums, zwischen mehreren Möglichkeiten zu wählen oder sich hin zur Selbstbestimmung zu entwickeln, sondern vielmehr liegt die Freiheit darin, Vernünftiges zu erkennen und es zu verwirklichen. Freiheit wird hier zur Notwendigkeit für das Subjekt, gemäß seinem vernünftigen Wesen zu handeln. Nach Hegel findet die Menschheit in der gesamten Menschheitsgeschichte hindurch zu einem Bewusstsein der Freiheit. Die Vernunft bildet die treibende Kraft und ist somit der Lenker und auch der Garant für einen guten Ausgang der Menschheitsgeschichte. Hegel und dessen Auffassung der Geschichte als eines kontinuierlichen Prozesses versteht aus aristotelischer Sicht das Allgemeine in der Geschichte immer nur verwirklicht durch das Besondere, andererseits, aus platonischer Sicht, ist dieses oder jenes Besondere bloß verwirklicht, indem es das Allgemeine als solches verwirklicht. In dieser Dialektik ist das Besondere aufgehoben im Allgemeinen und strebt seinem Ziel, dem Ende der Geschichte, als dem absoluten Fortschritt, 
mittels der Vernunft kontinuierlich entgegen (vgl. Hegel 1821/1969: 21-29). Es ist gerade nicht mehr das Individuelle, welchem ein wirkliches Sein zugesprochen wird, sondern vielmehr nur noch die vernunftgemäße Orientierung des Individuums am Allgemeinen, welcher Würdigung zukommt. In einem solchen Denken wird das Individuum als Mittel des Fortschritts verzweckt und der Fortschritt durch seine Zielgerichtetheit auf die Freiheit, eben die Notwendigkeit der Vernunft, legitimiert. In einem dialektischen Fortschrittsdenken nach Hegel liegt die Gefahr, selbst die katastrophalsten Ereignisse der Menschheitsgeschichte letztlich im Licht des unaufhörlichen Fortschritts der Freiheit zu betrachten. Die Extreme des Französischen Kaisertums führen zur Revolution und somit zum Fortschritt, die Schrecklichkeiten des Zweiten Weltkrieges zum friedlichen Nationalstaat und, in einer bestimmten Lesart der Marxschen Texte, die höchste Ausbeutung durch den Kapitalismus zur klassenlosen Gesellschaft. Jeder Schrecken der Geschichte bildet nach Hegelscher Auffassung ein notwendiges Durchgangsstadium für den darauffolgenden Fortschritt (vgl. Hegel 1969: 21-29). Für die Mobilisierung einer kritischen Masse bedeutet ein solches Denken, dass sie in gewisser Weise synchron mit dem Verlauf der Geschichte bleibt, d.h., sie kritisiert immer nur innerhalb und letztlich im Sinne der hegemonialen Strukturen. Besonders in einem Modell Hegelscher Geschichtsphilosophie liegt die Gefahr, dass die Bewegung der kritischen Masse letztlich zur Legitimation des von ihr kritisierten Gegenstands führt, weil eine kritische Masse in solch einem Paradigma notwendig die Versöhnung mit dem Gesamtprozess anstrebt. Es ist meiner Meinung nach nicht bestreitbar, dass nach geschichtlichen Katastrophen oftmals Prozesse beschleunigt werden, die in gewisser Weise zur Verbesserung der Lebenswelt geführt haben, aber der wesentliche Punkt ist, dass die ihr vorangegangenen Katastrophen nicht notwendig für eine Verbesserung der Lebenswelt waren.

Kurzum: Ich behaupte, dass die Mobilisierung einer kritischen Masse in einem Denkmodell des Fortschritts - zu diesem rechne ich einen vulgärmarxistischen Ansatz, welcher mit der Hegelschen Dialektik arbeitet - aus folgenden Gründen zum Scheitern verurteilt ist: Erstens versteht sich solch eine Mobilisierung, sei sie auch in Opposition zur restlichen Gesellschaft, letztlich doch immer in Einklang mit dem geschichtlichen Prozess und ist daher nicht wirklich kritisch. Zweitens versteht sich eine solche Mobilisierung aus den Idealen ihrer eigenen Gesellschaft heraus, sie reformiert
Vorhandenes und schafft somit nicht die bestehenden gesellschaftlichen Strukturen ab. In diesem Sinne wäre sie nicht ideologiekritisch. Drittens denkt eine Masse im Paradigma des Fortschritts nicht radikal kritisch, da der eigentliche Gegenstand der Kritik der Glaube einer Gesellschaft an den Fortschritt ist. Gerade dieser dritte Grund wirft eine Menge Fragen auf. Worin liegt denn die Bedingung der Möglichkeit der Mobilisierung einer kritischen Masse, wenn nicht in ihrem Glauben an den Fortschritt? Welches Paradigma eignet sich für ein Denken, in welchem sich eine kritische Masse mobilisieren lässt?

\section{Ein Konzept zur Mobilisierung einer kritischen Masse anhand der geschichtsphilosophischen Überlegungen von Walter Benjamin}

\subsection{Die Bedingung der Möglichkeit für die Mobilisierung einer kritischen Masse}

Worin also liegt die Bedingung für die Mobilisierung einer kritischen Masse? Worin sollte diese liegen, wenn sie nicht im Glauben an den Fortschritt begründet ist? Ein epistemologisches Fundament für eine kritische Lesart, und zugleich auch die Bedingung der Möglichkeit für die Mobilisierung einer kritischen Masse liegt, so behaupte ich, in den geschichtsphilosophischen Überlegungen von Walter Benjamin begründet. In diesem Modell fungiert Dialektik im Sinne einer prozessualen Auflösung von Gegensätzen nicht als prozessuale Denkfigur eines immanenten Geschichtssinns, sondern vielmehr als Stilllegung der prozessualen Denkfigur selbst, und somit wird ein Geschichtssinn überhaupt negiert. Die Erkenntnis liegt zu Beginn eines solchen Denkens darin, dass der Prozess und die ständige Bewegung der Geschichte nicht auf ein Ziel, nicht auf die Entfaltung der Freiheit angelegt ist, sondern vielmehr darin, dass die gesellschaftliche Handlungsund Sprachpraxis den Lebensraum der Individuen verdinglicht und zerstückelt bzw. zerstört.

Benjamin trennt in seinem sprachphilosophischen Essay Über Sprache überhaupt und über die Sprache des Menschen aus dem Jahre 1916 eine generell utopische Vorstellung absolut von dem Zustand der Gesellschaft. (Benjamin 1991/2003: 140-157) Zwar ist im Zustand der gegenwärtigen Gesellschaft die Möglichkeit einer Veränderung angelegt, aber nur indem absolut mit diesem Zustand gebrochen wird. Ein solches Denken verwehrt sich jeglichem reformistischen Gedanken. Im Zustand der Gesellschaft bleibt die Möglichkeit zum absoluten 
Bruch mit gerade diesem Zustand. In der gesellschaftlichen Handlungs- und Sprachpraxis, ja in ihrer äußersten Verdinglichung, ist selbst noch einmal das Moment ihrer Rettung, d.h. die Möglichkeit auf ein Jenseits der Verdinglichung in der Verdinglichung, angelegt. Genau darum braucht es kein Moment, in dem sich der gesellschaftlich immanente Gesamtzusammenhang transzendiert. Denn durch die totale Unterscheidung einer utopischen Vorstellung und der geschichtlichen Realität eröffnet sich dialektisch das Moment einer Möglichkeit des Eintretens des jeweiligen Gegenteils. Hierin unterscheidet sich die Benjaminsche Geschichtsphilosophie wesentlich von der Hegelschen. Während sich Freiheit in der Hegelschen Geschichtsphilosophie durch Vermittlung der sich überschreitenden Totalitäten innerhalb des geschichtlichen Prozesses selbst stets ein höheres Dasein schafft, eröffnet die Benjaminsche Geschichtsphilosophie gerade durch die Nichtvermittelbarkeit eine Möglichkeit darauf, den jeweiligen Gegensatz zu zerstören. Hegels Geschichtsphilosophie lebt von der Kontinuität der Vermittlung von Freiheit, die zu einer jeweils höheren affirmativen Daseinsform führt. Aus dieser Kontinuität leitet sich das Versprechen auf den guten Ausgang der Menschheitsgeschichte ab. Dieses Konzept einer Geschichtsphilosophie ist für Benjamins Auffassung gerade katastrophal, denn solch eine Ansicht führt unweigerlich zum Glauben an den Fortschritt. In diesem Konzept von Geschichtsphilosophie wird das Glück ständig vertagt, nicht das Jetzt ist in Hegelscher Geschichtsphilosophie entscheidend, sondern das Morgen. Der Bruch, die Unmöglichkeit der Vermittlung der Freiheit, und somit auch die Unmöglichkeit einer Identität von Individuum und Institution, wird nach Auffassung geschichtsphilosophischer Überlegungen von Benjamin gerade zur Bedingung der Möglichkeit für die Mobilisierung einer kritischen Masse.

$\mathrm{Zu}$ Beginn einer näheren Untersuchung der geschichtsphilosophischen Überlegungen von W. Benjamin möchte ich ein zentrales Zitat illustrieren, welches charakteristisch für das Geschichtsverständnis von Benjamin ist. Im Konvolut $N$ [Erkenntnistheoretisches, Theorie des Fortschritts] aus dem unvollendeten Passagen-Werk (1982) heißt es:

Nicht so ist es, daß das Vergangene sein Licht auf das Gegenwärtige oder das Gegenwärtige sein Licht auf das Vergangene wirft, sondern Bild ist dasjenige, worin das Gewesene mit dem Jetzt blitzhaft zu einer Konstellation zusammentritt. Mit anderen Worten: Bild ist Dialektik im Stillstand. Denn während die Beziehung der Gegen- wart zur Vergangenheit eine rein zeitliche, kontinuierliche ist, ist die des Gewesenen zum Jetzt dialektisch: ist nicht Verlauf, sondern Bild, sprunghaft (Benjamin 1991/2003: $576 f$.).

Nach und nach versuche ich, die Essenz dieser Zitate deutlich zu machen. Hierfür ist zuerst zu klären, was der Begriff Bild in den vorangegangenen Zitaten bedeutet. Diese Notwendigkeit ergibt sich im Rahmen dieses Aufsatzes für ein Verständnis der Methode der geschichtsphilosophischen Überlegungen nach Benjamin. Benjamin geht es nicht um Bilder aus der bildenden Kunst, so wie wir das in unserer Alltagssprache vielleicht vermuten könnten, sondern vielmehr bildet der Begriff Bild eine angemessene Beschreibung all dessen, was wir in unserer gesellschaftlichen Lebenswelt im Sinne der Kulturgüter vorfinden. Das Bild erscheint in Benjamins Denken als eine Konstruktion der Vergangenheit mit der Gegenwart, welche in der Form eines Textes in der Gegenwart ihren Ausdruck findet. Sigrid Weigel konstatiert in ihrer Arbeit Entstellte Ähnlichkeit zum Bildbegriff von Benjamin: „Mit seiner Theorie der Lesbarkeit und mit seiner Definition der dialektischen Bilder als gelesener betrachtet Benjamin Bilder nicht unter dem Aspekt der Repräsentation, sondern dem der Schrift: gleichsam als chiffrierte Verräumlichung oder aber als Konstellation“" (Weigel 1997: 52).

Der Begriff chiffrierte Verräumlichung meint, dass die Bilder immer schon in einem Raum bzw. in einem Deutungshorizont erscheinen, welcher einen Sinn für das Kollektiv und somit auch kollektive Identität selbst kommunizierbar macht. Die chiffrierte Verräumlichung ist der Bildraum. Bilder sind im Denken Benjamins Denkbilder bzw. Denkausschnitte, die als kulturelle Codes fungieren. Sigrid Weigel spezifiziert: „Seine Denkbilder sind gleichsam geschriebene dialektische Bilder, buchstäblich schrift-gewordene Konstellationen, in denen sich die Dialektik von Bild und Denken entfaltet und sichtbar wird" (ebd. 58). Das Bild als Denkausschnitt bildet einen erstarrten Denkfluss, der lesbar wird. Das Denkbild ist vorstellbar in Form eines tradierten Textes, der immer schon einen Sinn kommunizierbar macht, indem er in Konstellation zur Gegenwart und in Bezug zu anderen Texten bzw. Bildern steht. In den Denkbildern steckt die individuelle und die kollektive Erfahrung ununterscheidbar und doch unvereint. Als Denkbilder versteht sich u.a. die Kurzprosa, die wir bei Walter Benjamin selbst in Form vom Engel der Geschichte aus Über den Begriff der Geschichte (Benjamin 1991/2003: 302-304) oder 
dem bucklicht Männlein aus der Berliner Kindheit um Neunzehnhundert (vgl. Benjamin 1991/2003: 235-304) kennen. Diese Art der Denkbilder führt dazu, dass Geschichte nichts Festes, sondern etwas Progressives und Interpretatives bildet, und in Folge dessen zu der Annahme, dass es keine wesentliche Differenz zwischen einem prosaischen Text und der Geschichtsschreibung gibt, da beide Interpretationen, ja in gewisser Weise Konstruktionen der Welt und somit auch kollektiver Identitäten bilden. Diese Erkenntnis ist beängstigend und befreiend zugleich. Befreiend ist sie, denn sie entwirft eine völlig neue Topografie der Möglichkeiten das auszuhandeln, was kollektive Identität sein soll. Beängstigend ist sie, weil sie das Bewusstsein erweckt, dass Geschichte eine Geschichte der Sieger (Benjamin 1991/2003: 696) ist, denn gerade weil es kein plausibles Kriterium für die Geschichtsschreibung gibt, wird Macht ein Hauptkriterium. Es stellt sich nebenbei auch eine gewisse Orientierungslosigkeit innerhalb einer Gesellschaft angesichts der Entlarvung der Geschichte als bloßer Erzählung ein. Das gesellschaftliche Kollektiv ist dazu aufgefordert, die Welt in gewisser Weise $\mathrm{zu}$ erdichten. Friedrich Schlegel beschreibt dies treffend in seiner Transcendentalphilosophie: „Der Mensch dichtet gleichsam die Welt, nur weiß er es noch nicht“" (Schlegel 1801/1991: 105).

Geschichte bildet also nach Benjamin ein Konstrukt aus Denkbildern, die innerhalb eines Bildraums in eine kontinuierliche Ordnung gebracht werden. Das Problem liegt darin, die Bilder so zu erkennen, dass sich in ihnen der wahre Ausdruck des geschichtlichen Verlaufs offenbart. Was aber bedeutet das? Hierfür muss man sich von einem positiven Fortschrittsdenken distanzieren. Mauro Ponzi hält zusammenfassend in Bezugnahme auf den Begriff des Fortschritts gemäß eines Benjaminschen Verständnisses in seinem Artikel Topographie des Bildraums fest:

Der Begriff des Fortschritts setzt die Zerstörung des schon Existierenden voraus. Die ständige Veränderung der Moderne ist nur scheinbar eine Erneuerung, weil das Neue dazu bestimmt ist, von dem Neusten bald zerstört zu werden. Die Erneuerung der Moderne besteht eigentlich in der Wiederkehr des Immergleichen, und zwar in der Wiederholung des gleichen Zerstörungsschemas (Ponzi 2005: 112).

Das Wesen der Moderne ist somit permanente Zerstörung, und insofern das Individuum innerhalb einer Gesellschaft von dieser Zerstörung betroffen ist, ist die Situation der Moderne eine sozialpathologische. Das Wesen der Geschichte liegt nicht darin, ständig das Neuste hervorzubringen, sondern vielmehr darin, ständig das Vorhandene zu zerstören.

Der Bildbegriff Benjamins ist konstitutiv für eine Geschichtsphilosophie, die, im Gegensatz zur Hegelschen Geschichtsphilosophie, einen Zugang zur Geschichte ermöglicht, welcher die Geschichte nicht als einen Prozess des kontinuierlichen Fortschritts begreift. Dies gelingt, indem die Kontinuität der Vergangenheit zur Gegenwart in Frage gestellt wird. Diese Methode ist wesentlich für die Mobilisierung einer kritischen Masse.

Für die Mobilisierung einer kritischen Masse ist der Verzicht auf das Fortschrittsparadigma notwendig. Das Fortschrittsparadigma ist an eine bestimmte Auffassung von Geschichte und damit einhergehend an eine bestimmte geschichtsphilosophische Methode gebunden. Diese Methode besteht in der Beurteilung der Vergangenheit und ihrem Bezug zur Gegenwart. Will man also mit dem Fortschrittsparadigma brechen, um eine kritische Masse zu mobilisieren, so muss man bei der Beurteilung dessen anfangen, was man Vergangenheit nennt.

Die Vergangenheit ist uns nach Benjamin im Vergleich zu Hegel nicht in begrifflicher Weise als Geschichten im Sinne von Tatsachenberichten über die Geschichte greifbar, sondern, so heißt es im Konvolut $N$ [Erkenntnistheoretisches, Theorie des Fortschritts] im Passagen-Werk: „Geschichte zerfällt in Bilder, nicht in Geschichten“ (Benjamin 1991/2003: 565). Im letzten Fragment, unmittelbar vor seinem tragischen Tod im Jahr 1940, Über den Begriff der Geschichte (1940), schreibt Benjamin: „Nur als Bild, das auf Nimmerwiedersehen im Augenblick seiner Erkennbarkeit aufblitzt, ist die Vergangenheit festzuhalten." (Benjamin 1991/2003: 698). Unsere Lebenswelt besteht aus Bildern, in diesen Bildern tritt uns zugleich auch immer das Gewesene in unserer Gegenwart gegenüber. Diese Bilder sind zu verstehen als Kulturgüter aller Art, die es wie verschriftlichte Sprache zu lesen gilt, um sie auf das jeweilige Jetzt unserer Existenz zu beziehen. Das Gewesene erscheint in bildhafter Konstellation aus einem kulturellen Gut und der Gegenwart unserer Lebenswelt. Das Gewesene liegt nicht in einer Kette kontinuierlicher und kausaler Begriffe vor uns, sondern vielmehr tritt uns das Gewesene als heterogenes Diskontinuum in den Bildern unserer Lebenswelt entgegen. Genau diese Diskontinuität gilt es nach Benjamin augenblicklich zu erkennen. Es lässt sich in den Bildern unserer Kultur, den Bildern des Triumphes, zugleich der Verfall, die Katastrophe unserer Lebenswelt und der Geschichte 
erkennen. Diese alternative Möglichkeit, die Bilder des Triumphes als Bilder des Verfalls zu lesen, macht die Bilder zu dialektischen Bildern. Geschichte als ganze kann nur als reiner Verfall und als Katastrophe gedeutet und nicht kausal rekonstruiert werden. Rita Bischof vermerkt hier in ihrer Studie Teleskopagen, wahlweise. Der literarische Surrealismus und das Bild: „Dialektische Bilder lassen sich nicht zum System zusammenschließen, vielmehr ist die Ordnung, die sie begründen, eine unaufhebbar diskontinuierliche. Und diese verlangt nach einer anderen Beschreibungsstruktur, die ohne die Idee historischer Kausalität auskommt" (Bischof 2001: 364). Geschichte ist eine Geschichte kontinuierlicher Diskontinuität. Bilder fungieren als Monaden, indem jedes einzelne Bild aus unserer Lebenswelt als Bild des Verfalls unserer Lebenswelt gelesen werden kann. Bilder sind nicht kausal. Für ein Ursache-Wirkungsverhältnis bedeutet das: „Geschichte hat es nicht mit Entwicklungen, sondern mit Bildern $\mathrm{zu}$ tun, die untereinander ein Diskontinuum bilden, und das heißt, daß sie gerade nicht in ein Verhältnis von Ursache und Wirkung zueinander treten“" (Bischof 2001: 364). Während dialektisch-historische Kausalität im Sinne Hegels mit Notwendigkeit verfährt, ist im Ausdruck der sprachbildlichen Phänomene innerhalb des Bildraums das Moment einer Bedingung der Möglichkeit zum Bruch mit der nur scheinbar suggerierten Kontinuität im Sinne der dialektisch-historischen Kausalität Hegels angelegt. Rolf Tiedemann hält in seinen Studien zur Philosophie Walter Benjamins fest: „Der bildliche Ausdruck lässt sich so einfach nicht als solcher in den geschichtlichen Kontext einbetten: Er bricht das historische Kontinuum auseinander, zwingt es zum Stillstand und greift ein Bruchstück heraus“ (Tiedemann 1973: 154). Topografphisch gesehen stellen sich im jeweiligen Bildraum unserer konkret gesellschaftlichen Lebenswelt die Metaerzählungen dar, in denen die einzelnen Bilder für scheinbar zusammenhängende und kontinuierliche Zeichen des Fortschritts ausgegeben werden. Hingegen ist es der bildliche Ausdruck der einzelnen aus ihrem Kontext herausgesprengten Bilder, den Tiedemann meint. Dieser Ausdruck bringt eine sozialpathologische Dimension zum Vorschein, die es verbietet den Fortschritt zu denken.

Ich behaupte, dass diese alternative Lesart der gesellschaftlichen Lebensräume als Bilder des sozialpathologischen Verfalls, und somit die Abkehr von einem Paradigma des Fortschritts, eine notwendige Bedingung für die Möglichkeit zur Mobilisierung einer kritischen Masse bildet. Nur durch solch eine Lesart umgeht man ein ideologisches Geschichtsverständnis, welches von einem gesellschaftlichen Fortschritt ausgeht. Lediglich so ist Kritik ideologiekritisch, denn nur so tritt Kritik als wirklicher Fortschritt, wie es Adorno im Ausgangszitat dieses Aufsatzes feststellt, aus dem Bann heraus, auch aus dem des Fortschritts. Ich glaube, es gibt gute Gründe dafür zu sagen: Bleibt Kritik innerhalb des Fortschrittparadigmas, bleibt sie innerhalb der Ideologie. Man könnte sagen, das Fortschrittsparadigma bildet die Ideologie für jede Ideologie oder: das Fortschrittsparadigma ist die Bedingung für die Ideologie, sie ist Metaideologie. ${ }^{4}$

\subsection{Die Aufgabe einer kritischen Masse - Alternative Lesbarkeit und die Zerstörung der Bildräume}

An dieser Stelle sei noch einmal gerade das, für eine Betrachtungsweise im Sinne der Hegelschen Dialektik, seltsam anmutende Moment einer bildlichen, also einer Dialektik im Stillstand, betont. Dialektik im Benjaminschen Sinne ist nicht Prozess, sondern vielmehr stillgelegter Prozess. Aber woraus besteht diese Dialektik? Welche Größen treffen in solch einer Dialektik des Stillstands unversöhnlich aufeinander? Benjamin hierzu im Konvolut $N$ [Erkenntnistheoretisches, Theorie des Fortschritts] im Passagen-Werk: „[...] Bild ist dasjenige, worin das Gewesene mit dem Jetzt blitzhaft zu einer Konstellation zusammentritt" (Benjamin 1991/2003: 577). In Anbetracht der Hegelschen Dialektik gilt es immer danach zu fragen, wo diese Umschlagpunkte sind. Umschlagpunkte, Orte oder Zeitkontinua, an denen sich die jeweiligen Momente konkret geschichtlich zu höheren affirmativen Daseinsformen manifestieren. Einfacher gefragt: Wo und vor allem wann genau finden sich in der Menschheitsgeschichte die Schnittpunkte, an denen man aufzeigen kann, wie eine Epoche in einer anderen aufgehoben wird? Hegelsche Dialektik löst die Spannung zwischen

4 Selbstverständlich ist der Begriff der Ideologie im klassischen Sinne bei Marx weiter gefasst, aber trotzdem scheint sich sogar der Kerngedanke des Marxismus, wird er im Paradigma des Fortschritts gedacht, in Ideologie zu transformieren. Wird nämlich der Gedanke einer planwirtschaftlichen Ökonomie im Sinne des Sozialismus als fortschrittlicher Übergang aus der kapitalistischen Ökonomie gedacht, begibt man sich in das sozialdemokratische Paradigma eines reformistischen und demokratischen Sozialismus, welches nichtszu einem Ende des Kapitalismus, sondern zu seiner Erstarkung führt. 
den innergeschichtlichen Widersprüchen prozessual auf. Die Zeugnisse der vergangenen Epochen und ihre Forderungen an unsere Gegenwart werden mit dem gegenwärtigen Istzustand unserer Gesellschaft identifiziert. Das Vergangene ist identisch mit dem Gegenwärtigen, insofern das Vergangene im Gegenwärtigen in einer neuen und höheren Daseinsform behauptet wird. In Benjaminscher Dialektik hingegen trifft das Gewesene als das Vergangene blitzhaft mit dem Jetzt zusammen, aber in einer nicht mehr vermittelbaren Widersprüchlichkeit. Hier regiert reine Unvereinbarkeit von Vergangenheit und Gegenwart, die bis zur äußersten Spannung im dialektischen Bild zum Ausdruck gelangt. Diese stillgelegte Unvereinbarkeit ermöglicht eine auf die Gegenwart gerichtete Erkenntnis. Ansgar Hillach vermerkt in seinem Aufsatz Dialektisches Bild hierzu: „Wirklichkeit, die Wirklichkeit der dialektischen Geschichts- als Gegenwartserkenntnis, findet Benjamin im Zeitdifferential, in der kleinsten, blitzhaft erhellenden Zeitdifferenz des Jetzt von dem Jetzt, das aufscheint in einem Zitat oder Zeugnis der Vergangenheit." (Hillach 2000: 191). Das kleinste Zeitdifferenztial, das Erkenntnis immer zu überwinden hat, ist also die Differenz des Augenblicks des Jetzt der Erkenntnis und des Jetzt im Bild, welches erkannt wird. Genaugenommen ist das Jetzt, welches Gegenstand der Erkenntnis ist, schon im Jetzt der Erkenntnis das jüngst Vergangene, aber im Jetzt der Erkennbarkeit eröffnet die Erkenntnis dem jüngst Vergangenen einen Handlungsraum, der es ermöglicht aus der katastrophalen Struktur der Gesellschaft auszusteigen. Dieses Aussteigen bildet das Moment einer revolutionären Handlung.

Um zu verdeutlichen, was Benjamin meint, nennt er die Gegenwart das Jetzt und die Vergangenheit das Gewesene. Das Gewesene ist für Benjamin, im Gegensatz zum Begriff des Vergangenen, nicht abgeschlossen, vielmehr ist es präsent in den Bildern und Symbolen einer Gesellschaft. Im Bild als Produkt der Gesellschaft ist, so könnte man sagen, zwar verschleiert, dessen ganzer Entstehungsprozess enthalten. Es geht Benjamin aber nicht darum, diesen Prozess zu rekonstruieren, sondern vielmehr unter der erscheinenden Oberfläche den Ausdruck seiner wahren Geschichtlichkeit freizulegen. Die einzelnen Bilder, welche als Zitate aus der Vergangenheit im Jetzt erscheinen, künden nur noch von der leidvollen Vergangenheit der Benachteiligten aller vorangegangenen Epochen. Die Vergangenheit findet somit keinen Anknüpfungspunkt, keinen kontinuierlichen Anschluss mehr an die Gegenwart. Vergangenheit ist gegenwärtig im jeweils gegenwärtigen Jetzt und erweist sich als nicht mehr vereinbar mit diesem Jetzt und als nicht mehr vereinbar mit dem gesamten Geschichtsverlauf. Bilder des Triumphes werden nach Benjamin in seinem Text Über den Begriff der Geschichte (1940) zu Bildern der Barbarei (Benjamin 1991/2003: 701). Vergangenheit wird in einer jetztzeitlichen Erfahrung zum Ankläger der Gegenwart. Das mit der Vergangenheit (im Sinne des Gewesenen) aufgeladene Bild im Jetzt als herausgesprengtes, verhält sich zum Kontext, dem übrigen Bildraum der Gesellschaft, in einer dialektisch unaufhebbaren Heterogenität. Diese Heterogenität wiederum bildet einen Ort, eine Leerstelle, in welcher es möglich ist, den Lauf der Geschichte als Geschichte der Unterdrückung durch die bürgerliche Geschichtsschreibung zum Stillstand zu bringen. Führt ein aus seinem Kontext heraus gesprengtes Bild zum Stillstand der konkreten Verhältnisse, dann offenbart sich der dialektische Charakter des Bildes nicht nur im Hinblick auf den übrigen Bildraum, sondern auch im Hinblick auf die wirkliche Lebenswelt.

Das Bild als dialektisches zerstört den übrigen Bildraum der Gegenwart, insofern die jetztzeitliche Erkenntnis eines Bildes den sozialpathologischen Charakters des gesamten Bildraums zum Ausdruck bringt. Die kritische Betrachterin und der kritische Betrachter erkennen durch den dystopischen Charakter der Gesellschaft, dass das Glück nicht vertagt, sondern vielmehr niemals eintreten wird. Somit ermöglicht sich durch eine Stilllegung einer hegemonialen Konstruktion von Geschichte, durch einen Bruch mit der Geschichtsschreibung, die Mobilisierung und die Handlungsweise einer kritischen Masse. Mit Benjamin gesprochen, sprengt diese Masse durch ihr konkretes Handeln ein Loch in das Kontinuum des homogenen Zeitverlaufs, d. h., die Masse bricht mit der Metaerzählung, die im Bann des Fortschritts steht. Indem die kritische Masse das Traumbild des Fortschritts, das Bild der Identität von Individuum und Institution, von einzelnem Bürger und objektivem Geist, in ein Bild der Heterogenität, ein Bild der totalen Nichtversöhnbarkeit und Nichtidentität, ein Bild der Katastrophe umdeutet, entlarvt die kritische Masse den Charakter der Geschichte als eine Konstruktion der bürgerlichen Gesellschaft. Die Vergangenheit im Sinne des Gewesenen wird zur Jetztzeit, und in dieser wird Geschichte umgedeutet. Heiner Weidemann merkt in seinem Aufsatz Traum/ Erwachen an: „Das Jetzt der Erkennbarkeit bleibt der Erkenntnis wie dem Erkennenden eingeschrieben. Dabei wird das Damals als das erkannt, was nur jetzt so erkannt werden kann und dieses Jetzt konstituiert 
sich als der Moment, in dem jenes Damals zu erkennen ist." (Weidemann 2000: 343). Dieses Zitat erscheint im Zusammenhang mit einer Bemerkung Benjamins aus dem Konvolut N [Erkenntnistheoretisches, Theorie des Fortschritts] aus dem Passagen-Werk:

Die kopernikanische Wendung in der geschichtlichen Anschauung ist diese: man hielt für den fixen Punkt das Gewesene und sah die Gegenwart bemüht, an dieses Feste die Erkenntnis tastend heranzuführen. Nun soll sich dieses Verhältnis umkehren und das Gewesene zum dialektischen Umschlag zum Einfall des erwachten Bewußtseins werden (Benjamin 1991/2003: 49of.).

Das Gewesene im Sinne der Vergangenheit bildet nichts Festes und Unveränderliches, sondern trägt an die Gegenwart eine Option. In der Umdeutung des Verhältnisses von Vergangenheit und Gegenwart kristallisiert sich ein Bewusstsein für die Notwendigkeit einer revolutionären Handlung. Dies geschieht, indem das Gewesene aus seinem Kontext herausgerissen und als Bild in der Jetztzeit zitiert wird. Indem das Gewesene als Monument der Vergangenheit gegenwärtig als Trugbild der Gesellschaft von der kritischen Betrachterin und dem kritischen Betrachter als kritischer Historikerin und kritischem Historiker gedeutet wird, entpuppt sich das Gewesene im Sinne des gegenwärtigen Trugbilds als trojanisches Pferd. Als trojanisches Pferd entpuppt sich das Gewesene in solch einer Lesart, weil das Gewesene nicht zur Legitimation des Gegenwärtigen, sondern vielmehr zu dessen Anklage führt. Somit ist das Damals, das Gewesene im Jetzt des Erkenntnisakts, zu reiner Aktualität geworden. Kopernikanisch daran ist, dass die Geschichtserkenntnis nicht ein Betrachten einer fixierten und festen Vergangenheit in der Gegenwart ist, sondern dass das Gewesene sich in jedem Jetzt aufs Neue erkennbar machen kann. Was erkannt wird, ist aber somit die wesentliche Relevanz des Gewesenen im Jetzt und für das Jetzt, und somit wird überhaupt Gegenwärtiges, wird überhaupt Jetzt erkannt. Der Sinn der Geschichte als solcher ist immer nur im Jetzt für das Jetzt und niemals als archivierte Vergangenheit abrufbar, die Geschichte posthum einen Sinn verleihen möchte. Dieser Sinn definiert sich durch die Erfahrung einer reinen Sinnlosigkeit gegenwärtiger Existenz innerhalb einer Gesellschaft. Norbert Bolz vermerkt in seinem Aufsatz Bedingungen der Möglichkeit historischer Erfahrungen: „Das heißt aber nicht, daß das Bild eine zeitliche Beziehung der Gegenwart zur Vergangenheit stiftet, sondern vielmehr eine dialektisch-sprunghafte des Gewesenen zum Jetzt" (Bolz 1991: 154). Erst diese sprunghafte Erkenntnis der Sinnlosigkeit, die sich im Bildraum als gesellschaftliche Pathologie darstellt, stiftet den Sinn einer kritischen Masse; dieser liegt nicht im Streben nach Fortschritt, sondern vielmehr in der tatsächlichen Unterbrechung der Katastrophe begründet. Wenn der Bildraum zerstört ist und der Verlauf der Geschichte im Sinne der Geschichtsschreibung unterbrochen ist, besteht die Möglichkeit, Geschichte neu zu gestalten, erst dann ist Fortschritt denkbar.

Bolz weiter in seinem Aufsatz Bedingungen der Möglichkeit historischer Erfahrungen: „Der Historiker hat es nicht mit Menschen und Kulturwerken, sondern mit Skeletten und Monaden zu tun“ (Bolz 1991: 156). Aber was bedeutet diese Aussage von Norbert Bolz? Benjamin vermerkt in seinen ersten Notizen Pariser Passagen im Passagen-Werk: "All das hat nie gelebt: so wahr nie ein Skelett gelebt hat, sondern nur ein Mensch“ (Benjamin 1991/2003: 1000). Die Vergangenheit, welche das Fundament und die Legitimation unserer sogenannten Fortschrittsgesellschaft begründet, ist nichts weiter als eine tote und konstruierte Fassade. Die Bilder und Symbole sind nicht Kulturwerke, Werke des Triumphes, sondern Ruinen, und als solche spiegeln sie wie Monaden die gesamte Menschheitsgeschichte als einzige Ruine, als Katastrophe wider. Das Skelett ist tot und jeder einzelne Knochen an ihm lässt es als totes erscheinen. Benjamin stellt sich auf die Seite des marxistischen Diktums der Entfremdung, indem er feststellt, dass die Straßen, die Gebäude und all die sichtbaren Strukturen unserer Lebenswelt bloß durch den Menschen gedacht werden können. Sie existieren nur, indem sie Produkte qua menschlicher Arbeit und gesellschaftlicher Interaktion sind. Da sie Produkte des Menschen sind, fungieren sie als Text, der lesbar geworden ist, und wie ein Skelett die anatomische Struktur des Menschen aufweist, weisen die Strukturen unserer Lebenswelt Spuren menschlicher Arbeit auf. Das Lebendige an diesen Strukturen ist der Mensch. Somit erscheinen die Strukturen aber zugleich als solche bloß als tote, weil das Menschliche aus ihnen getilgt ist. Diese Erkenntnis aber, dass die Strukturen als tote gelesen werden, lässt den Menschen nicht mehr bloß als den Strukturen unterworfenen erscheinen, sondern weist ihn vielmehr als den Schöpfer der Strukturen aus und attestiert ihm zugleich die Macht, mit diesen Strukturen zu brechen. Ebenso wie im Bauch des hölzernen Pferdes gemäß des Mythos die griechischen Soldaten versteckt waren, so stecken auch in den einzelnen Kulturgütern, welche die Strukturen unserer Lebenswelt bilden, die Spuren menschlicher Arbeit, welche die Kulturgüter für sich 
genommen als Skelett des Menschen und somit als tote ausweisen.

\subsection{Die geschichtsphilosophischen Überle- gungen von Walter Benjamin anhand eines Beispiels}

Nach den, zugegebenermaßen, etwas komplizierten Illustrationen der Methoden der geschichtsphilosophischen Überlegungen von Walter Benjamin soll ein Beispiel für die konkrete Anwendung der Methoden gegeben werden. Anhand dieses Beispiels soll aufgezeigt werden, wie sich eine kritische Masse gegen ihre Verhältnisse und gegen Unterdrückung mobilisieren lässt. Benjamin selbst hat uns in seinem Fragment Über den Begriff der Geschichte (1940) ein historisches Beispiel seiner Methode hinterlassen:

Die Geschichte ist Gegenstand einer Konstruktion, deren Ort nicht die homogene und leere Zeit, sondern die von Jetztzeit erfüllte bildet. So war für Robespierre das Antike Rom eine mit Jetztzeit geladene Vergangenheit, die er aus dem Kontinuum der Geschichte heraussprengte. Die Französische Revolution verstand sich als wiedergekehrtes Rom. Sie zitierte das alte Rom genau wie die Mode eine vergangene Tracht zitiert (Benjamin 1991/2003: 701).

Rom, welches als Bild der Legitimation für das französische Kaisertum diente, wird aus dem Kontext gesprengt und als ein Zitat erkannt, welches Wesentliches für das Jetzt in sich birgt. Somit wird das Jetzt erkannt, und die Mechanismen der Unterdrückung und deren Schein werden entlarvt durch eine neue Konstruktion, eine neue Lesart, in welcher das Gewesene, das alte Rom, in eine neue Konstellation gebracht wird. Das antike Rom stiftet in Konstellation mit dem Jetzt in der Lesart Robespierres keine Identität mehr in Bezugnahme auf das französische Kaisertum. Für Robespierre war es eine augenblickliche Erkenntnis, dass das antike Rom nicht mehr der Legitimation des französischen Kaiserreichs diente. Dies führte dazu, dass das antike Rom als Bild letztlich keine Identität zwischen Kaiser und Volk stiften konnte. Diese Spannung zerriss den gesamten Bildraum, die gesamte Metaerzählung des französischen Kaisertums. Diese Konstellation sprengt den Bildraum der Gesellschaft als solchen. Die ganze Geschichtsschreibung wird als bloßer Mythos abgetan. Im Jetzt der Erkennbarkeit setzt das Zitieren des antiken Roms im Jetzt das Jetzt in eine kritische Lage, in der die Möglichkeit auf eine revolutionäre Handlung besteht. Somit wird ein Bild der Unterdrückung zu einem Bild der Emanzipation.
Das antike Rom dient nun als Bild der Befreiung von den Unterdrückern. Es entsteht eine Abkehr von einem bürgerlichen Verständnis des antiken Roms, in welchem Kaiser wie Nero als Galionsfiguren herhalten mussten. Neue Identifikationsfiguren des alten Roms wie Spartakus werden zu Zeiten der Revolution zitiert.

Die Bilder, seien sie auch vorerst Trugbilder, erscheinen uns im Bildraum unserer sich wirklich ereignenden Geschichte. Es gilt, diese Wirklichkeit wie einen Text zu lesen, und dies kann man durchaus im wörtlichen Sinne verstehen. Nimmt man beispielsweise einen Roman, ein Schulbuch oder ein anderes Werk zur Hand, dann hat man es in gewisser Weise auch immer mit dem Lebenswerk des Autors und somit auch mit der Epoche, in welcher der Autor tätig war, zu tun. Das kleinste Schriftstück, das kleinste Zitat aus der Vergangenheit, bringt mit sich, zwar verborgen, seine ganze Epoche. Es gilt Benjamin, jedes Kulturgut, jedes Sprachbild, als Text im Sinne eines Sprachphänomens scharf zu machen, zu zünden und damit die gesamte Geschichte zu sprengen. Aufbewahrt und aufgehoben findet sich der gesamte Geschichtsverlauf nicht einfach so in den Bildern, meint, der Verlauf der Geschichte liegt nicht einfach offen da, sondern ist verborgen in den Bildern und Texten als möglicher Erkenntnis des Jetzt. Somit sind die Trugbilder zugleich auch immer der Möglichkeit nach Bilder der Emanzipation und Bilder des Erwachens aus dem Schlummer des Fortschrittdenkens. Dies ermöglicht ihr dialektischen Charakter. Erst wenn keine Differenz zwischen dem Bildraum und dem Leib des geschichtlichen Subjekts mehr vorhanden ist, dann ist der Bildraum zum Leibraum geworden.

\subsection{Das Konzept des Leibraums}

Was macht den Leibraum zum Leibraum? Mit dem vorangegangenen Beispiel von Benjamin ist klarer geworden, wie die Mobilisierung einer kritischen Masse sich ereignen könnte, aber was genau macht die Lesart in Benjamins Beispiel zum Leibraum und nicht wiederum zur Ideologie oder zum Trugbild? Es bleibt uns an dieser Stelle nicht erspart, einen längeren Textabschnitt

5 Ähnliches geschieht später in der Sowjetunion und anderen sozialistischen Staaten. Es werden hier beispielsweise Sportvereine gegründet, welche den Namen, Spartakus tragen, um eine Identifikationsgröße gegen den „,bürgerlichen Sport" innerhalb der Sportgemeinschaft für das Proletariat zu stiften. Das berühmteste Beispiel hierfür ist wohl der 1922 gegründete Sportverein Spartak Moskau. 
zu betrachten, der uns vor Augen führt, was Benjamin unter dem Begriff Leibraum versteht. Zu Ende seines berühmten Aufsatzes Der Surrealismus. Die letzte Momentaufnahme der europäischen Intelligenz umschreibt Benjamin den Leibraum wie folgt:

Denn auch im Witz, in der Beschimpfung, im Mißverständnis, überall, wo ein Handeln selber das Bild aus sich herausstellt und ist, in sich hineinreißt und frißt, wo die Nähe sich selbst aus den Augen sieht, tut dieser gesuchte Bildraum sich auf, die Welt allseitiger und integraler Aktualität, in der die gute Stube ausfällt, der Raum mit einem Wort, in welchem der politische Materialismus und die physische Kreatur den inneren Menschen, die Psyche, das Individuum oder was sonst wir ihnen vorwerfen wollen, nach dialektischer Gerechtigkeit, so daß kein Glied ihm unzerrißen bleibt, miteinander teilen. Dennoch aber - ja gerade nach solch dialektischer Vernichtung - wird dieser Raum noch Bildraum, und konkreter Leibraum sein (Benjamin 1991/2003: 309).

Sowohl das Bild als auch der Bildraum stehen als Ausdruck in einem Verhältnis der Mittelbarkeit, sie sind mittelbarer Ausdruck des gesellschaftlichen Kollektivs. Diese Mittelbarkeit kennzeichnet eine Distanz bzw. eine Differenz des Ausgedrückten zum Auszudrückenden. Auf der einen Seite braucht es nach Benjamin ein Moment des Innehaltens, um das Bild als solches zu identifizieren. Dies geschieht eben durch Formen wie Witz, Beschimpfung und Mißverständnis. Auf der anderen Seite bezeichnen alle drei Formen eine Unmittelbarkeit, in welcher aktual eine Verschiebung der Bilder durch die unmittelbare Partizipation sich vollzieht. Diese Partizipation geht unmittelbar in das Bild selbst ein, in welchem sich der Witz, die Beschimpfung oder das Mißverständnis ausdrückt. Das Bild fungiert somit nicht als ein identitätsstiftendes, sondern vielmehr drückt sich unmittelbar die inkommensurable Identität selbst im Bild aus. Somit ist Handeln selber das Bild. Dieses Handeln eben ist es, welches das Bild in sich hineinreißt und frißt, wo die Nähe sich selbst aus den Augen sieht, tut dieser gesuchte Bildraum sich auf, die Welt allseitiger und integraler Aktualität. Keine Differenz steht mehr zwischen dem Bild und dem, was es ausdrücken soll. Es existiert nur noch integrale Aktualität, in welcher das Bild und das, was es ausdrücken soll, ununterscheidbar bleiben. Die gute Stube bezeichnet die Differenz zwischen Ausgedrücktem und Ausdruck, die nun ausfällt. Dieser schöpferischen Unmittelbarkeit der unmittelbaren Teilhabe wohnt aber ihr zerstörerisches Moment inne. Denn es bleibt dem Bildraum kein Glied unzerrißen. Erst die Zerstörung des vorhandenen
Bildes durch einen heroischen Akt der Dekonstruktion wandelt die determinierte Topologie gesellschaftlicher Identitäten, welche der Bildraum darstellt, in eine Topographie der Nichtidentität, die die Bedingung der Möglichkeit authentischer, dem leiblichen Ausdruck des gesellschaftlichen Kollektivs angemessener Identitätssuche bildet. Der Bildraum, an dem sich unsere individuelle Identität orientiert, ist unserer (leiblichen) Existenz nicht angemessen. Eben darauf verweist Adorno, wenn er in seinem berühmten Zitat konstatiert: „Leben, das Sinn hätte, fragte nicht danach“ (Adorno: 1997, 369). Sinnvolles Leben hat unmittelbar Sinn, und somit muss die Frage nach Sinn in einem sinnvollen Leben gerade ausfallen. Sinn vollzieht sich dann unmittelbar in der Sinnsuche im Vollzug des eigenen Lebens. Dies schließt nicht die Reflexion aus, sondern bindet sie unmittelbar an den Vollzug des gesellschaftlichen Lebens. Sigrid Weigel fasst dies in ihrer Arbeit Entstellte Ähnlichkeit. Walter Benjamins theoretische Schreibweise so zusammen: „In diesem Bildraum [...] sind Distanz und Grenze zwischen Subjekt und Bild aufgehoben, insofern das Subjekt selbst in den Bildraum eingegangen ist, indem es selbst, mit seinem Leib, an ihm teilhat" (Weigel 1997: 116). Diesen Ausdruck zu entdecken und sich durch ihn vom trügerischen Schein des existierenden Bildraums hin zu einem dem Leib angemessenen Bildraum, einem Leibraum, zu emanzipieren, ist die bleibende Aufgabe des gesellschaftlichen Kollektivs, doch vorerst Aufgabe der marxistischen Intellektuellen. Durch diese Entdeckung führen die marxistischen Intellektuellen $\mathrm{zu}$ einer Mobilisierung einer kritischen Masse. Der wesentliche Punkt des Konzepts des Leibraums aber, so behaupte ich, liegt darin, dass ihm ein materialistisches Axiom zugrunde liegt. Der Leibraum ist der Ausdruck der wirklichen Verhältnisse, in denen die Menschen leben. Der Leibraum drückt unmittelbar das ganze Elend und die Armut aus, zu der ein Großteil der Menschheit verdammt ist, aber auch die Ausbeutung, die damit verbunden ist. Für die Tätigkeit einer kritischen Masse gilt also, die Bilder und Symbole aus ihrem verheerenden Kontext zu sprengen und diese auf eine angemessene Art zu deuten. Durch dieses Deuten wird der Bildraum zerstört und in einen Leibraum, in welchem die materiellen Verhältnisse und deren prekäre Situation zum Ausdruck kommen, umgewandelt. Dieser Leibraum selbst ist der Ort einer neuen Geschichtsschreibung, einer Geschichtsschreibung der Unterdrückten. Die Schrift bildet in diesem Konzept der Geschichtsschreibung den ausgebeuteten Leib des Kollektivs. Dem Bild als dialektischem wohnt 
die Möglichkeit inne, in einer Form erkannt zu werden, welche unmittelbar den Ausdruck des Leibes sichtbar macht. Aber wo und wie lassen sich dialektische Bilder als solche erkennen?

\subsection{Die Funktion der dialektischen Bilder}

Der dialektische Charakter der Bilder, wird er erkannt, bildet die Möglichkeit einer Mobilisierung der kritischen Masse und in Folge dessen der Aneignung der Gegenwart. Benjamin sagt im Konvolut N [Erkenntnistheoretisches, Theorie des Fortschritts] in seinem Passagen-Werk (1982) über das dialektische Bild: „Das dialektische Bild ist ein aufblitzendes. So, als ein im Jetzt der Erkennbarkeit aufblitzendes Bild, ist das Gewesene festzuhalten. Die Rettung, die dergestalt und nur dergestalt vollzogen wird, läßt immer nur an dem, im nächsten Augenblick schon unrettbar Verlorenen sich vollziehen“ (Benjamin 1991/2003: 592). Das, was im Jetzt gerettet wird, ist nicht bloß das Gewesene, sondern das Gewesene für das Jetzt. Das Gewesene als im Jetzt Anwesendes wird nur in diesem Sinne gerettet, weil der Sinn des Gewesenen auf das, worauf es im Jetzt verweist, erkannt werden kann. Dieser Moment aber ist im nächsten Augenblick unrettbar verloren. Nur wenn das, worauf das Gewesene im Jetzt verweist, nicht nur erkannt wird, sondern die Erkenntnis unmittelbar in eine politische Handlung übergeht, geschieht Rettung im eigentlichen Sinne. Um es konkret geschichtlich im Hinblick auf Benjamins eigene Biografie auf den Punkt zu bringen: Rettung vollzieht sich immer als Rettung vor dem Faschismus. Die Bilder gehören alle einer bestimmten Zeit an. Der Begriff der Rettung suggeriert, dass wir in einem permanenten Ausnahmezustand leben. Der Ausnahmezustand ist der Normalfall, und dieser bildet die permanente Katastrophe. Rettung meint in diesem Sinne keine religiöse oder mystische Kategorie, sondern die bloße Rettung der leiblichen Existenz. Es geht darum, mit dem Leben davonzukommen, doch die Erkenntnis, die bei Benjamin zur Rettung führen kann, ist nur vorübergehend. In dem Fragment Über den Begriff der Geschichte (1940) heißt es: „Der historische Index der Bilder sagt nämlich nicht nur, daß sie einer bestimmten Zeit angehören, er sagt vor allem, dass sie erst in einer bestimmten Zeit zur Lesbarkeit kommen“ (Benjamin 1991/2003: 703). Dinge kommen zu ihrer Lesbarkeit $\mathrm{zu}$ einer jeweils bestimmten Zeit. Lesbarkeit meint, dass sie erkannt werden. Diese Zeit kann eine sein, in welcher das Erkannte in gewisser Weise gar nicht mehr wirklich da ist. In dem Moment, in dem die Dinge verfallen, werden sie erkannt. So verhält es sich auch mit der Passage de l'Opéra. Diese Passage ist dem Tod geweiht, d.h. sie soll abgerissen werden. In ihr ist nur noch Rest und Abfall, der darauf wartet, entsorgt zu werden. Aber genau dieses Alte und Verweste kommt im Jetzt zu seiner Erkennbarkeit, indem es Wesentliches über die Jetztzeit, die gegenwärtige Epoche, in der es erkannt wird, verrät. In den Passagen ereignen sich Bilder, die sich in dieser Weise nicht so einfach ereignen würden. Warum? Weil die Dinge in den Passagen willkürlich in Konstellation gebracht werden. Man muss sich das wie bei einem Umzug vorstellen, wenn man alle Dinge verpackt, um sie mit ins neue Heim zu nehmen. Denn dann fragt man sich bei vielen einzelnen Dingen oftmals, ob man sie überhaupt mitnehmen oder ob man sie besser entsorgen soll. Man betrachtet die Dinge dann oft in einem ganz anderen Licht. Beim Packen sortiert man die Dinge für den Transport neu. Da steht beispielsweise schon mal ein Topf bei den Büchern. Es ereignen sich dadurch neue Konstellationen. Für Benjamin, der stets am Detail orientiert ist, ist somit die Passage ein Ort des Traumes, und dieser Ort bietet gleichzeitig die Möglichkeit des Erwachens. Die Möglichkeit des Erwachens ist in den Passagen höher als an anderen Orten, denn in ihnen stehen die Dinge in seltsamen Verbindungen. Dies tun sie zum einen, weil die Passage ohnehin allerlei verschiedene Geschäfte nebeneinander beherbergt, zum anderen, weil die Passage de l'Opéra vor ihrem Abriss steht. Viele Geschäfte sind schon geschlossen und überall stehen Kisten mit verschiedensten Dingen, die entsorgt werden müssen oder an einen anderen Ort sollen. Benjamin beschreibt diese Passage in einem längeren Abschnitt in seinen frühen Entwürfen Pariser Passagen II im PassagenWerk (1982) wie folgt:

Solch ein ideales Panorama einer kaum verflossenen Urzeit tut mit dem Blick durch die in alle Städte verteilten Passagen sich auf. Hier haust der letzte Dinosaurus Europas, der Konsument. An diesen Höhlenwänden wuchert als unvordenkliche Flora die Ware und geht, wie die Gewebe in Geschwüren die, die regellosesten Verbindungen ein. Einer Welt geheimer Affinitäten: Palme und Staubwedel, Föhnapparat und die Venus von Milo, Prothese und Briefsteller finden sich hier, wie nach langer Trennung zusammen. Lauernd lagert die Odaliske neben dem Tintenfaß. Adorantinnen heben Aschbecher wie Opferschalen. Diese Auslagen sind ein Rebus und es liegt einem auf der Zunge, wie hier das Vogelfutter in der Fixierschale einer Dunkelkammer verwahrt wird, Blumensamen neben dem 
Feldstecher, die abgebrochenen Schrauben auf dem Notenheft und der Revolver überm Goldfischglas zu lesen sind. Übrigens sieht nichts von alldem neu aus. Die Goldfische stammen vielleicht aus einem inzwischen lange versiegten Bassin, der Revolver wird corpus delicti gewesen sein, und sicherlich haben diese Noten ihre frühere Besitzerin vom Hungertode bewahren können, als die letzten Eleven fortblieben (Benjamin 1991/2003: 1045f.).

An diesem Beispiel werden mehrere wichtige Punkte der Benjaminschen Erkenntnistheorie sichtbar. Zuallererst betont Benjamin, dass sich durch die Passagen im Allgemeinen ein Blick auf ein ideales Panorama einer kaum verflossenen Urzeit auftut. Schon hier wird klar, dass die Erfahrung in den Passagen das Kontinuum der Zeit im Sinne der dialektischen Bilder sprengt. In ihnen wird die urzeitlich-utopische Vorstellung einer Welt ohne Ausbeutung im Modus des Vermissens, d.h. ihres Ausbleibens, erfahren. Die Spuren, die uns über die Erfahrung in die Urzeit führen, sind Spuren der Sozialpathologie. Des Weiteren erwähnt Benjamin die verschiedensten Dinge, die willkürlich in Konstellation zueinander stehen. Sie sind somit aus ihrem Kontext herausgerissen. Der Briefsteller steht nicht im Schreibwarensortiment, sondern er taucht in geheimer Affinität mit der Prothese auf. Es sind die geheimen Affinitäten, die Benjamin als magische Korrespondenzen versteht. In den Passagen macht man Erfahrungen mit der Sprache, die es nur in der Kindheit gab, denn hier existiert nur das Spiel mit den (Sprach-)Bildern der eigenen und der kollektiven Erfahrung. All diese Bilder in Konstellation sind dialektische, weil sie die Möglichkeit bieten, als erwachtes Bewusstsein auf das Jetzt bezogen zu werden und in den Traum einzufallen. Am Ende des Abschnitts blitzt sogar kurz das Erwachen hindurch, wenn es heißt: „Die Goldfische stammen vielleicht aus einem inzwischen lange versiegten Bassin, der Revolver wird corpus delicti gewesen sein, und sicherlich haben diese Noten ihre frühere Besitzerin vom Hungertode bewahren können, als die letzten Eleven fortblieben“ (Benjamin 1991/2003: 1045f.). Hier deutet Benjamin die Bilder (Goldfisch, Revolver, Noten) als dialektische, indem er sie pathologisch und im Sinne einer kritischen Lesart deutet. Erst hier werden die Bilder des Kollektivs wirklich zu dialektischen Bildern, welche eine andere Lesart erlauben. In den Passagen fällt dies leichter, weil die Dinge nicht in einer logischen Ordnung zueinander stehen, und somit fällt das Detail leichter ins Auge. Hier offenbart die Ware ihren wahren Charakter. Über die Passage heißt es: „Zu beiden Seiten dieser Gänge [...] laufen die elegantesten Warenläden hin, so daß eine solche Passage eine Stadt, eine Welt im kleinen ist [...] “ (Benjamin 1991/2003: 1044). Die Passage selbst hat monadologische Qualitäten, sie bildet den gesamten Bildraum der Gesellschaft in verkürzter Art und Weise ab. „Die Passagen (das sind die Galerien, die in ihr vergangenes Dasein führen) [...]“ (Benjamin 1991/2003: 1046). Dieses vergangene Dasein erscheint uns im dialektischen Bild, dort erscheint uns das Gewesene in der Gegenwart. Dieses Gewesene kennzeichnet sich durch den Missstand der Gegenwart als einer versäumten Gegenwart ohne Ausbeutung.

\section{Offene Probleme einer Geschichtsphilosophie nach Walter Benjamin in Bezug auf eine kritische Masse}

Was ist nun das Fazit für die Mobilisierung einer kritischen Masse? Erstens scheint es wesentlich, dass die Bedingung der Möglichkeit der Mobilisierung einer kritischen Masse selbstständiges Denken ist. Selbstständiges Denken meint hier, in der Lage zu sein, verschiedene Eindrücke und Denkbilder in neue Zusammenhänge $\mathrm{zu}$ bringen, so wie Benjamin die Dinge und somit auch die gesamte Lebenswelt auf ihren Verfall hin deutet. Zweitens lassen sich kritische Massen dort mobilisieren, wo das Bewusstsein der Aussichtslosigkeit, um mit Benjamin zu sprechen, das Bewusstsein der Katastrophe, entsteht. Eine Masse ist nicht wirklich kritisch, wenn sie sich beispielsweise für mehr Kindergeld, bessere Schulen oder Frauenrechte mobilisieren lässt, sondern erst dann, wenn sie versteht, dass es nichts und niemanden gibt, der ihr Rechenschaft und Befriedigung sichern kann, außer sie selbst. Es geht für die kritische Masse nicht darum, auszuhandeln, wie man sich besser oder humaner beherrschen lassen kann, sondern selbst mit der Geschichte der Herrschaft zu brechen, um die eigene Geschichte zu schreiben. Der Prozess einer neuen Geschichtsschreibung im Sinne einer Bestimmung der Gegenwart geht von einzelnen Individuen aus und greift im Idealfall über auf die kritische Masse. Hier liegt wohl auch das problematische Moment der Mobilisierung einer kritischen Masse, denn die Erkenntnis einzelner Intellektueller, dass die Geschichte im Sinne der bürgerlichen Geschichtsschreibung angesichts der Katastrophe unterbrochen werden muss, ist nutzlos, wenn sie nicht umgesetzt werden kann. Dies gelingt aber eben nur, indem die kritische Masse mobilisiert werden kann, nur sie kann die Katastrophe unterbrechen. Ist aber die Mobilisierung einer kritischen Masse und ihr 
Erwachen zu einem emanzipatorischen Bewusstsein überhaupt möglich? In Bezugnahme auf Benjamin und seine Zeit kann man sagen, dass das Erwachen ein paar Intellektuellen und anderen wenigen Ausnahmen gelungen ist. Bernd Witte legt in seinem Essay Paris Berlin - Paris. Zum Zusammenhang von individueller, literarischer und gesellschaftlicher Erfahrung in Walter Benjamins Spätwerk den Finger in die Wunde von Benjamins Problem: „Das Erwachen aus dem Kollektiv des neunzehnten Jahrhunderts, [...] erweist sich schließlich als Wunschbild eines Einzelnen, der vom kollektiven Subjekt der Geschichte im Stich gelassen wurde“ (Witte 1984: 26). Der Epoche als solcher ist ein Erwachen nicht gelungen. Es scheint, als sei ein Erwachen bis heute nicht gelungen. Die Bilder unserer Zivilisation werden immer noch in einem Paradigma der Moderne einem Fortschrittsdenken unterworfen. Die Traumbilder haben noch nicht ihre dialektische Kraft entfaltet. Es liegt wohl aber auch daran, dass der Theorie-PraxisZusammenhang im Marxschen Sinne doch, zum Leid der gesamten Menschheit, (noch) nicht ganz der Wirklichkeit entspricht. Leider ist unsere Lebenswelt paradox, in ihr führt die Erkenntnis des Richtigen nicht immer notwendig zu einem richtigen Handeln. Was das Erwachen betrifft, ist Benjamin an anderer Stelle selbst sehr skeptisch. Gelingt dieses Erwachen? Benjamin bringt dies in der Berliner Kindheit um Neunzehnhundert im Abschnitt Der Mond zur Geltung:

Der Mond, der am Himmel gestanden hatte, war plötzlich immer schneller angewachsen. Näher und näher kommend riß er den Planeten auseinander. Das Geländer des eisernen Balkons, auf dem wir alle über der Straße Platz genommen hatten, zerfiel in Stücke, und die Leiber, die ihn bevölkert hatten, bröckelten geschwind nach allen Seiten auseinander. Der Trichter, den der Mond im Kommen bildete, sog alles in sich hinein. Nichts konnte hoffen, unverwandelt durch ihn hindurchzugehen. Wenn es jetzt Schmerz gibt, gibt es keinen Gott, hörte ich mich erkennen, und sammelte zugleich, was ich hinübernehmen wollte. Alles tat ich in einen Vers. Er war mein Abschied. O Stern und Blume, Geist und Kleid, Lieb, Leid und Zeit und Ewigkeit! Jedoch, indem ich diesen Worten mich anheimzugeben suchte, war ich schon erwacht. Und nun erst schien das Grauen, mit dem eben der Mond mich überzogen hatte, sich auf ewig, trostlos, bei mir einzunisten. Denn dies Erwachen steckte nicht, wie andere, dem Traum sein Ziel, sondern verriet mir, daß es ihm entgangen und das Regiment des Mondes, welches ich als Kind erfahren hatte, für eine weitere Weltzeit gescheitert war (Benjamin 1991/2003: 203).
Benjamins Erwachen ist ein böses. Die Erkenntnis, die aus diesem Erwachen resultiert, ist die, dass er, Walter Benjamin als marxistischer Intellektueller, feststellen muss, dass niemand erwachen wird und der Albtraum des Faschismus weitergeträumt wird. Die Massen werden ihren Preis zahlen müssen.

Aber was passiert, wenn eine kritische Masse sich mobilisiert? Ist nicht die NSDAP in ihren Anfängen auch eine Mobilisierung von kritischer Masse gegenüber einer sterbenden Weimarer Republik? Stehen nicht auch die fremdenfeindlichen Positionen der AfD oder der FPÖ im Kontext einer kritischen Masse? Dies lässt sich zumindest mit Benjamins Konzept nicht denken, denn dadurch, dass der Leibraum beansprucht, die wirklichen und dies meint die materiellen Verhältnisse der Individuen und der Gesellschaft, in der sie leben, zum Ausdruck zu bringen, ist eine Mobilisierung unter diesem Banner als solche notwendig emanzipatorisch. Allerdings, und dies geschieht durchaus sehr häufig, liegt die Gefahr darin, dass die Lösung einer erkannten materiellen Armut in ein Konzept umschlägt, welches die materielle Ebene wieder verlässt. So auch religiös fundamentalistische Ideologien, die auf die Armut und Ausbeutung bestimmter Gruppen verweisen, um sie für einen Kampf gegen die „Ungläubigen“ zu mobilisieren. Demgegenüber bleibt zu sagen, dass das Benjaminsche Konzept des Leibraums die materielle Ebene nicht verlässt, sondern durch die Erkenntnis einer katastrophalen Gegenwart materieller Verhältnisse auf deren Veränderung und gerechten Verteilung für alle Menschen besteht und eben nicht abschweift in eine ideologische Weltflucht. Die NSDAP und eben auch der IS verzweckt nur die materielle Armut der Massen für ihre ideologischen Konzepte. Ihr Ziel ist es nicht, die Herrschaft der Masse zu gewährleisten und auf lange Sicht den Wohlstand und die Entwicklung der gesamten Menschheit zu fördern, sondern die Massen für bestimmte Zwecke $\mathrm{zu}$ unterwerfen. Doch muss eingestanden werden, dass selbst die Russische Revolution einem totalitären Schicksal nicht entging. In ihr wurden Zeichen des Triumphes, Zeichen der Orthodoxie und der Zaren zu Zeichen der Unterdrückung und Zeichen der Unterdrückung, Zeichen des bäuerlichen Lebens und der Arbeit zu Zeichen des Triumphes. Doch die Zeichen des bäuerlichen Lebens und der Arbeit wurden selbst wiederum ein Werkzeug der Unterdrückung. Davon zeugt die Ära des Stalinismus. Doch vielleicht ist das Risiko jeder emanzipatorischen Bewegung in totalitärer Vernichtung zu enden niemals auszublenden. Es 
steht nur die Entscheidung aus, ob wir in Anbetracht dieser Gefahr das Risiko auf uns nehmen und handeln oder zum Unrecht schweigen. Ich bevorzuge es $\mathrm{zu}$ handeln.

Es bleibt übrig darüber nachzudenken, wie konkrete Handlungen einer kritischen Masse im Sinne eines geschichtsphilosophischen Konzepts nach Benjamin aussehen könnten. Eine Lehre könnte es sein, den Respekt vor der Vergangenheit und die Angst vor der Zukunft zu verlieren und aus dieser Kraft heraus die Gegenwart als solche abzulehnen. Aber wie genau? Vielleicht verbietet es sich in gewisser Weise darüber nachzudenken, was nach dem radikalen Bruch steht, denn der Bruch will ja das ganz Andere, das angesichts unserer Geschichte völlig Undenkbare, nämlich eine Gegenwart ohne Herrschaft und Ausbeutung. Ich denke, es geht vielmehr um eine Haltung als um eine konkrete Handlungsanweisung. Konkret beobachten wir immer öfter, wie Menschen, die aus purer Not und Verfolgung flüchten, wie Kriege und politische Krisen unsere Lebenswelt immer mehr erschüttern und wo die Ausbeutung des Menschen durch den Menschen immer bestialischere Züge annimmt. Ist es nicht vielleicht an der Zeit, die Ursachen der Kriege, der Gewalt und der Ausbeutung auf unser System zu beziehen? Benjamin erinnert uns an die Möglichkeit, mit dem ganzen System zu brechen, die konstruierte Geschichte der Sieger, ihre Metaerzählung, ihren Bildraum zu löschen, um neu zu beginnen mit dem Bewusstsein zu scheitern. Doch auch in Anbetracht einer Niederlage bleibt das Verlangen, eine eigene Geschichte zu schreiben, eine Geschichte der Unterdrückten. Die Haltung also, die aus dem geschichtsphilosophischen Konzept von Benjamin nur resultieren kann, ist eine unversöhnliche Haltung der kritischen Masse gegenüber dem System und Misstrauen in jeden Kompromiss. Für die anderen gilt Trotzkis Fluch aus der Verbannung: Nein, in diesen beschränkten, konservativen und feigen Köpfen findet unser Zeitalter keinen Raum (Trotzki 1962: 108).
Bischof, R. (2001): Teleskopagen, wahlweise. Der literarische Surrealismus und das Bild. In: Heftrich, E. (Hg.): Das Abendland. Forschungen zur Geschichte europäischen Geisteslebens, 29. Frankfurt am Main: Klostermann.

Bolz, N. (1984): Bedingungen der Möglichkeit historischer Erfahrungen. In: Derselbe (Hg.): Passagen. Walter Benjamins Urgeschichte des neunzehnten Jahrhunderts. Frankfurt am Main: Wilhelm Fink Verlag. 136-162.

Canetti, E. (1994): Masse und Macht. In: Derselbe: Gesammelte Werke, III. München: Hanser.

Hegel, G.W.F. (1969): Vorlesungen über die Philosophie der Geschichte. In: Derselbe: Gesammelte Schriften, XII, Moldenhauer, E. (Hg.), Michel, K.M. (Hg.), Frankfurt am Main: Suhrkamp.

Hillach, A. (200o): Dialektisches Bild. In: Opitz, M. (Hg.)/ Wizisla, E. (Hg.): Benjamins Begriffe, Band I. Frankfurt am Main: Suhrkamp, 186-229.

Horkheimer, M. (1937/1988): Traditionelle und Kritische Theorie. In: Derselbe: Gesammelte Schriften, IV. Herausgegeben von Schmidt, A. (Hg.)/Schmid Noerr, G. (Hg.). Frankfurt am Main: Fischer, 163-216.

Ponzi, M. (2005): Topographie des Bildraums. In: Witte B. (Hg.): Topographien der Erinnerung. $\mathrm{Zu}$ Walter Benjamins Passagen. Berlin: Könighausen Neumann, 121-146.

Schlegel, F. (1988): Friedrich Schlegel. Kritische Ausgabe seiner Werke (KFSA), Behler, E. (Hg), Paderborn: Ferdinand Schöningh.

Schlegel, F. (1801/1991): Transcendentalphilosophie. Eingeleitet und mit Erläuterungen versehen von Michael Elsässer. Hamburg: Felix Meiner Verlag.

Tiedemann, R. (1973): Studien zur Philosophie Walter Benjamins. Frankfurt am Main: Suhrkamp.

Trotzki, L. (1935/1962): Tagebuch im Exil. Essen: Mehring.

Weidemann, H. (2000): Traum/Erwachen. In: Opitz, M. (Hg)/Wizisla, E. (Hg): Benjamins Begriffe, Band I. Frankfurt am Main: Suhrkamp, 342-411.

Weigel, S. (1997): Entstellte Ähnlichkeit. Walter Benjamins theoretische Schreibweise. Frankfurt am Main: Fischer.

Witte, B. (1984): Paris - Berlin - Paris. Zum Zusammenhang von individueller, literarischer und gesellschaftlicher Erfahrung in Walter Benjamins Spätwerk. In: Bolz, N. (Hg.)/Witte, B. (Hg.): Passagen. Walter Benjamins Urgeschichte des XIX. Jahrhunderts. München: Fink Verlag, $17-26$.

\section{Literatur}

Adorno, W. T. (1975/2016): Fortschritt. In: Bulthaup, P.: Materialien zu Benjamins Thesen über den Begriff der Geschichte. Beiträge und Interpretationen. Frankfurt am Main: Suhrkamp, 149-169.

Benjamin, W. (1991/2003): Gesammelte Schriften (unter Mitwirkung von: Adorno, T. W., Sholem, G.), Tiedemann, R. (Hg.)/Schweppenhäuser, H. (Hg.). Frankfurt am Main: Suhrkamp. 